\title{
Focused Ion Beam-based Three Dimensional Analysis of Optic Nerve Axons
}

\author{
Avishai Amir *, Yin, Xinghau**, Trapp Bruce D.** and Kidd, Grahame J.**
}

*Department of Materials Science and Engineering, Case Western Reserve University, 10900 Euclid Ave, Cleveland, OH, 44106

**Department of Neurosciences, Lerner Research Institute, Cleveland Clinic Foundation, Cleveland, Ohio 44120

Axonal dysfunction and degeneration underlie many CNS diseases, including those affecting the myelin sheath in individuals with multiple sclerosis [1]. Optic nerves are an attractive model system for studying CNS axonal dysfunction because they are anatomically accessible and the axons are homogeneous in size and function. Microscopy-based assessment of axonal diameters, myelin internodal length, and distribution of organelles such as mitochondria is difficult, however, because optic nerve axons are small $(0.5-2 \mu \mathrm{m})$, densely packed, and often follow sinuous courses along the nerve.

A recent paper described a method for providing serial TEM-like images using a dual focused ion beam (FIB) to mill the sample and SEM to image the block face sections using backscattered electrons [2]. This method offers several advantages for imaging optic nerves, including automated data collection, data sets imaging axons in three dimensions, and the ability to prescan the tissue to locate areas of interest; in this case areas in which optic nerve fibers were favorably oriented. Potential difficulties for optic nerve examination have also been noted, including contribution of underlying dense myelin to the backscatter image due to the primary electron beam's interaction volume. This could be a significant obstacle in nerves with substantial myelin disruption and axonal damage.

To test whether this dual beam approach was an effective approach for analyzing rodent optic nerve preparations, we studied samples from $\mathrm{P} 0$-CNS mice, a model animal in which genetic alteration of myelin produces substantial axonal pathology in older mice [3]. In TEM samples, tannic acid enhances contrast of proteins and so we tested whether tannic acid pretreatment might reduce the impact of lipid rich myelin in our images.

Three P0-CNS mice aged 6 months were perfusion fixed for EM with 2.5\% glutaraldehyde and 4\% paraformaldehyde in Sorrensen's phosphate buffer as previously described [3]. Following the method of Knott et al [2], the nerves were washed in buffer and one was treated with $1 \%$ tannic acid for $1 \mathrm{hr}$. All samples were then washed in buffer, treated $1 \mathrm{hr}$ in buffered $0.4 \%$ osmium tetroxide, washed and stained in ethanolic uranyl acetate, dehydrated and embedded in Durcupan resin. Blocks were trimmed to expose tissue and sputter coated with $300 \mathrm{~nm}$ palladium. Tissues were examined in a Nova Nanolab-200 Dual Beam FIB, (FEI) system at the Swagelok Center for Surface Analysis of Materials at Case Western Reserve University. The section cutting was performed with a $1 \mathrm{nA}$ ion beam current and imaged in high-resolution mode (immersion lens) using the through lens detector in backscattered electron mode. The images were acquired in a high resolution format of 2048-1768 pixels and scanning time of $0.1 \mathrm{msec}$ per line [2]. Images were viewed, registered, corrected for aspect ratio, and structures manually or automatically segmented using Image $J$ software. 
Images of optic nerves without tannic acid provided good resolution of axons and myelin, and organelles such as mitochondria were well resolved in axons. Series of 70 images at $\sim 100 \mathrm{~nm}$ steps at $\times 5000$ were generated, which took $18 \mathrm{hrs}$ of milling and scanning. At this magnification, viewing fields covering $50 \mu \mathrm{m}$ of optic nerve were generated (Fig 1A). Such data sets included $\sim 25$ axons that could be traced for $20-50 \mu \mathrm{m}$ in three dimensions. Datasets could be reliced to provide cross sections of axons (Fig 1B). Organelles such as mitochondria were readily visible at this magnification (Fig 1C) and manual and semiautomated tracing provided 3D distributions of mitochondria in axons. Addition of the tannic acid preparative step greatly enhanced the contrast of axonal mitochondria and microtubules (Fig 1D), and provided greater ultrastructural detail within regions of the myelin such as paranodal loops. Some difficulties were encountered, as mentioned by Knott et al [2], including occasional curtaining of the block face during automated sectioning. Image registration between slices was also drifted, with shifts of 5-10 $\mu \mathrm{m}$ encountered within a data set. This was easily corrected using ImageJ software posthoc. Some less-pronounced drift within the images due to the long acquisition time was also occasionally observed which could not be easily corrected and may constrain precision of volumetric calculations were absolute volumes required. Disparity in the slice thickness was also a factor, although this could be potentially corrected for by using internal fudicials to correctly position the ion beam patterns before every slice.

We conclude that the dual beam slice and view approach offers an unparallel tool for analyzing and quantifying axonal structure, myelin organization, and organelle distributions in optic nerve axons and other CNS tissues. Staining for this purpose is enhanced by tannic acid pretreatment.

References:

[1] Trapp B.D. and Nave. K-H. Annual Review of Neuroscience, 2008, 31: 247

[2] Knott et al, The Journal of Neuroscience, March 19, (2008) • 28(12):2959.

[3] Yin et al., The Journal of Neuroscience, Jan 30 (2006). 72: 469.

[4] The aid of Paul Anzalone FEI Company is gratefully acknowledged.
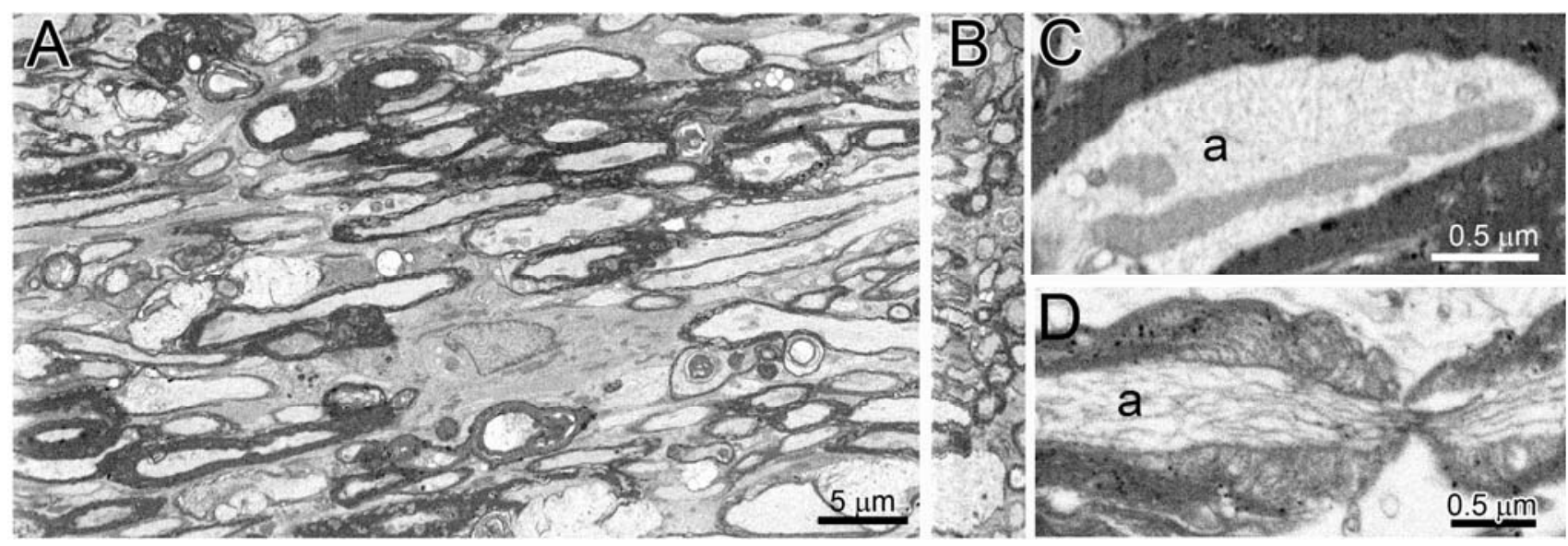

Figure 1 Axons imaged by scan-and-slice dual beam FIB approach. A. First slice of series. Note that the axonal and myelin pathology are a feature of this sample. B. End views showing fibers in cross section were generated by software reslicing from the dataset of 70 slices (scale same as in A). C. In routine preparations, mitochondria were readily resolved in the axon (a) but addition of tannic acid treatment (D) enhanced contrast of microtubules, neurofilaments, and some paranodal structure. 